\title{
Drawing up a collaborative contract: Amino acid cross-feeding between inter-species bacterial pairs
}

\author{
Erin Kelly ${ }^{1}$, Alexandria Fischer ${ }^{1}$, and Cynthia Collins ${ }^{1}$ \\ ${ }^{1}$ Rensselaer Polytechnic Institute
}

December 20, 2020

\begin{abstract}
Synthetic microbial communities have the potential to enable new platforms for bioproduction of biofuels and biopharmaceuticals. However, using engineered communities is often assumed to be difficult because of anticipated challenges in establishing and controlling community composition. Cross-feeding between microbial auxotrophs has the potential to facilitate co-culture growth and stability through a mutualistic ecological interaction. We assessed cross-feeding between 13 Escherichia coli amino acid auxotrophs paired with a leucine auxotroph of Bacillus megaterium. We developed a minimal media capable of supporting the growth of both bacteria and used the media to study co-culture growth of the 13 interspecies pairs of auxotrophs in batch and continuous culture, and on semi-solid media. In batch culture, eight of thirteen pairs of auxotrophs were observed to grow in co-culture. We developed a new metric to quantify the impact of cross-feeding on co-culture growth. Six pairs also showed long-term stability in continuous culture, where co-culture growth at different dilution rates highlighted differences in cross-feeding amongst the pairs. Finally, we found that cross-feeding-dependent growth on semi-solid media is highly stringent and enables identification of the most efficient pairs. These results demonstrate that cross-feeding is a viable approach for controlling community composition within diverse synthetic communities.
\end{abstract}

\section{Hosted file}

Drawingupacollaborativecontract.pdf available at https://authorea.com/users/383989/articles/ 499803-drawing-up-a-collaborative-contract-amino-acid-cross-feeding-between-interspecies-bacterial-pairs 


\begin{tabular}{|c|c|c|}
\hline A & M9 salts (M) & B3 salts (M) \\
\hline $\mathrm{Na}_{2} \mathrm{HPO}_{4}$ & $4.8 \times 10^{-2}$ & - \\
\hline $\mathrm{KH}_{2} \mathrm{PO}_{4}$ & $2.2 \times 10^{-2}$ & $1.8 \times 10^{-2}$ \\
\hline $\mathrm{K}_{2} \mathrm{HPO}_{4}$ & - & $1.4 \times 10^{-2}$ \\
\hline $\mathrm{NH}_{4} \mathrm{Cl}$ & $1.9 \times 10^{-1}$ & - \\
\hline$\left(\mathrm{NH}_{4}\right)_{2} \mathrm{HPO}_{4}$ & - & $6.2 \times 10^{-3}$ \\
\hline $\mathrm{NaCl}$ & $8.6 \times 10^{-3}$ & - \\
\hline $\mathrm{MgSO}_{4}$ & $2.0 \times 10^{-3}$ & $1.7 \times 10^{-3}$ \\
\hline $\mathrm{CaCl}_{2}$ & $1.0 \times 10^{-4}$ & - \\
\hline $\mathrm{FeSO}_{4}$ & - & $3.6 \times 10^{-5}$ \\
\hline $\mathrm{MnSO}_{4}$ & - & $4.1 \times 10^{-5}$ \\
\hline
\end{tabular}
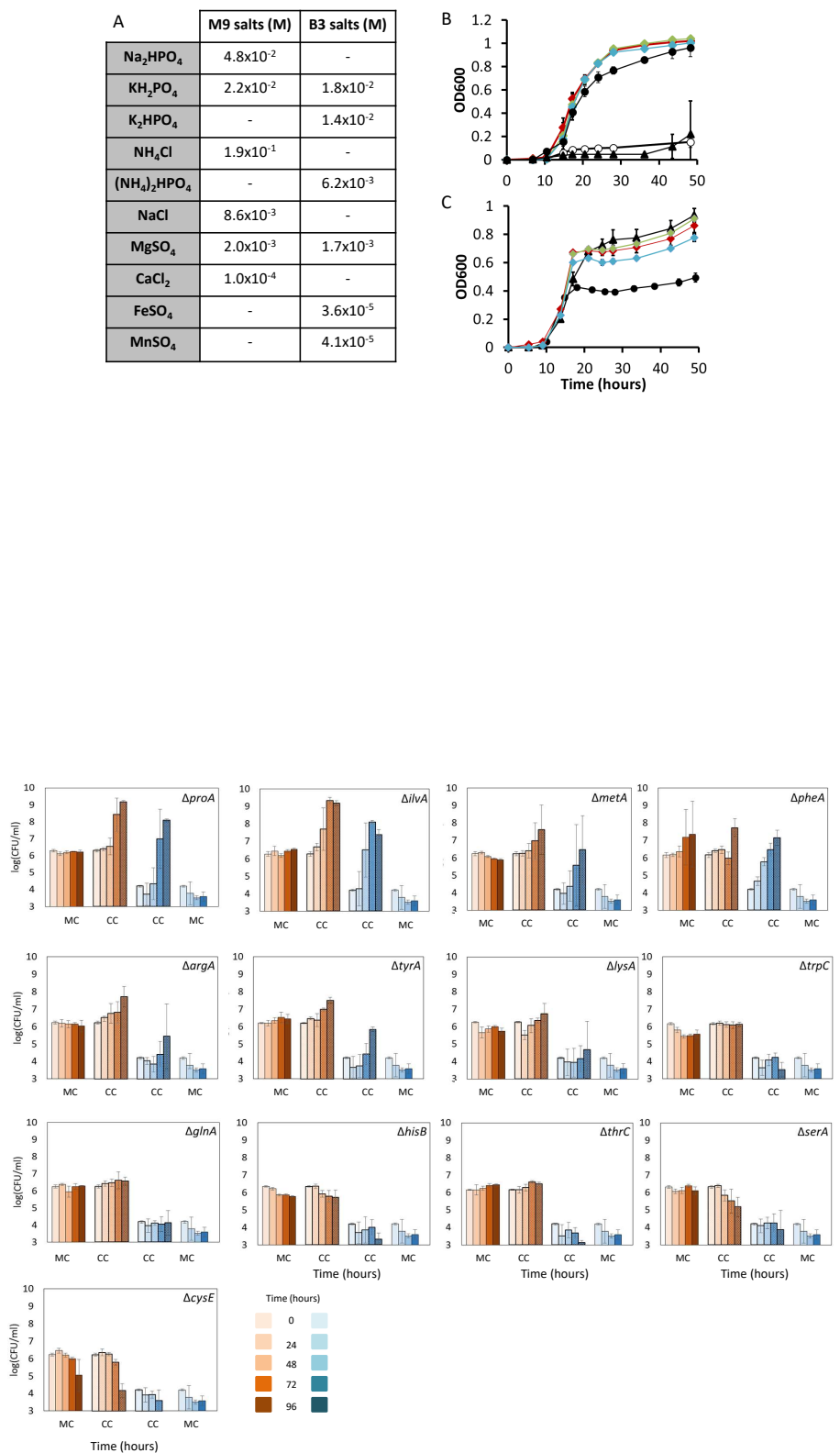

Time (hours)

[1] ${ }^{0}$

24
${ }^{24}$
${ }^{48}$
72 
A B. megaterium:E. coli

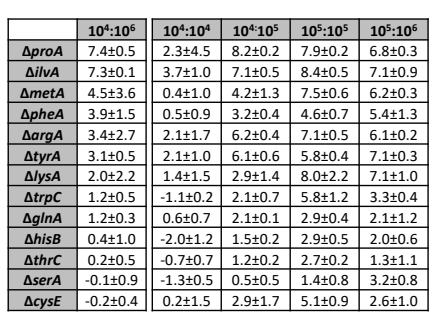

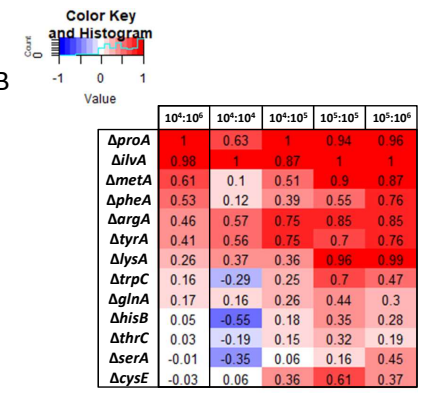
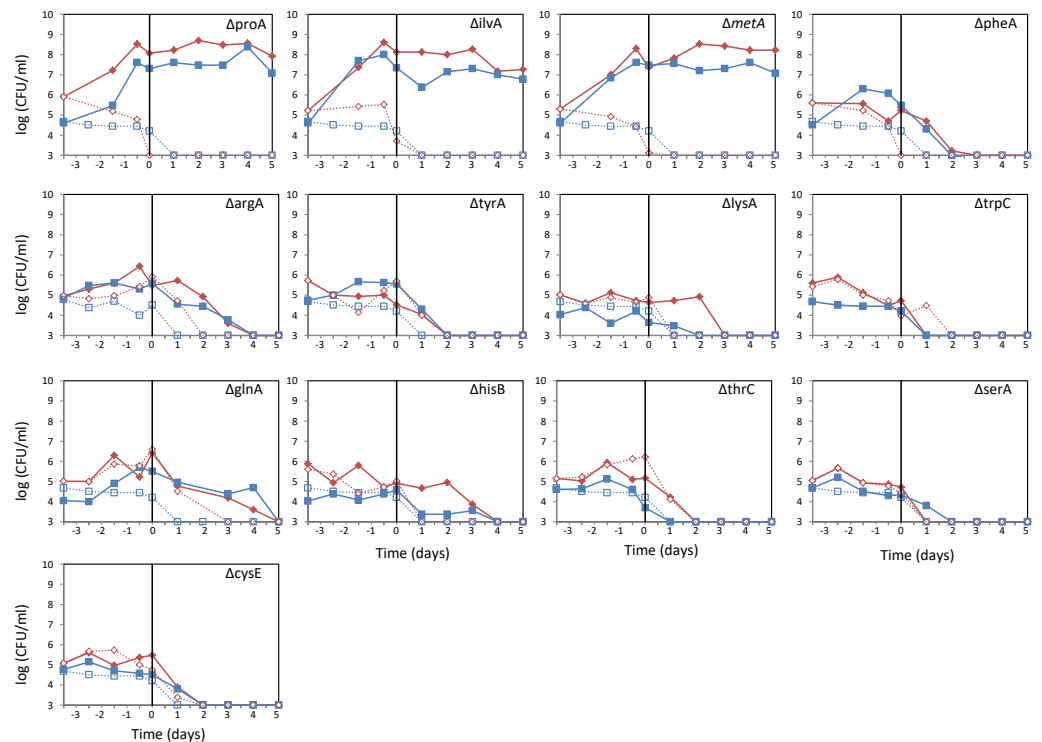

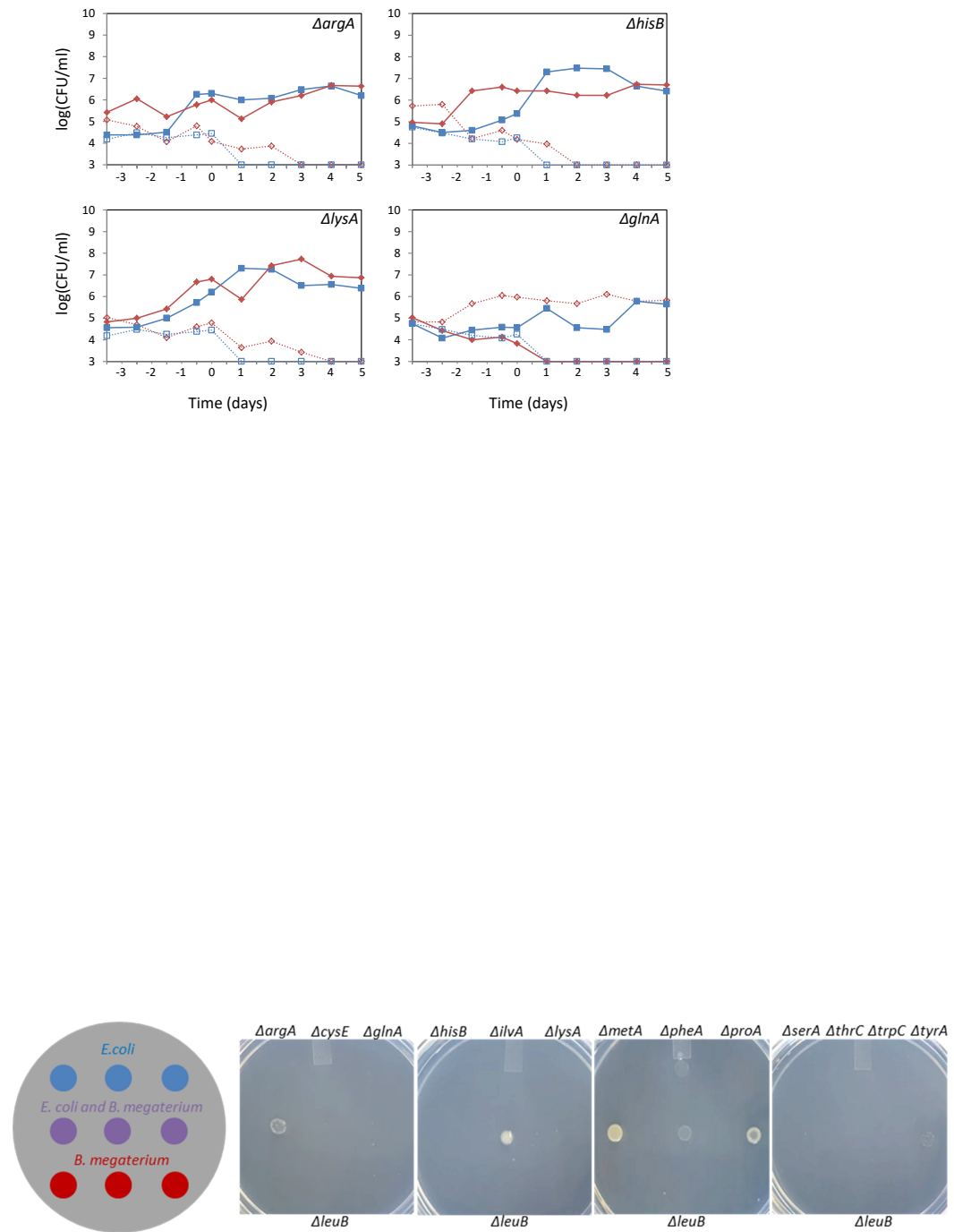